\title{
The Pig Food Intake Measurement System Based on TinyOS and WSN
}

\author{
Shuai Wang ${ }^{1}$, Yun $\mathrm{GaO}^{1}$, Xuan $\mathrm{Li}^{1}$, Minggang Lei ${ }^{2}$, Kun Chen ${ }^{1}$, Tianyang \\ Chen $^{1}$, Shipeng Tian $^{1}$, Yanliang Guo ${ }^{1}$ \\ ${ }^{1}$ College of Engineering, Huazhong Agricultural University, Wuhan, Hubei, 430070 \\ ${ }^{2}$ The Cooperative Innovation Center for Sustainable Pig Production, Wuhan, hubei, 430070 China \\ hunter2011@foxmail.com
}

\section{Keywords: TinyOS; Wireless Sensor Network; Real-time Measurement; Food Intake Amount}

\begin{abstract}
Now, we are unable to make pig feed intake real-time monitoring and prevention of swine diseases factors, this paper designed a pig food intake measurement system based on TinyOS and wireless sensor networks. The system can realize intelligent pig, real-time monitoring through wireless nodes intake of pigs, and can send data to the gateway via a wireless sensor network, and then transmitted to the remote computer, the pig food intake data with the data in the database will take for comparison, analysis of the health of pigs, to achieve disease prevention. Thus, this has a very important significance for Chinese modern pig feed industry, pig standardization, improving productivity, and promoting the development of China's pig farming industry
\end{abstract}

\section{Introduction}

Pig food intake is to determine the animal growth and the production of an important economic factor. In recent years, along with large-scale development of aquaculture and stocking density continues to increase, all kinds of livestock and poultry epidemic breaking out in a huge disaster for the aquaculture industry, but also a threat to human life and health. Animal welfare more and more attention, not only affects the quality of welfare of livestock products, and also affect the export of animal products, especially in pig farming, feed intake of pigs directly determine the survival rate of pigs and pork quality [2]. So we designed a set based on TinyOS wireless sensor networks and pig food intake measurement system for real-time monitoring of intake of pigs, and pigs intake system analysis, forecasting pig health. The system can intelligently monitor pig food intake, improve the conditions for pig farming, improve production efficiency, scientific analysis can observe the health of pigs, thereby effectively preventing disease, for the development of the intelligent pig has significance .

\section{System Structure}

This project is to provide a measure of the amount of TinyOS-based pig feeding systems and wireless sensor networks. Including wireless sensor network consists of two parts as well as PC. Wireless sensor network includes a two-part sensor node network consists of nodes in pigs and the environment as well as the sensor base. A PC or network server, resolve locally installed software, databases, IIS, etc. Local administrators can manage the entire system, remote users can access the site through a browser, it has permission to obtain sensor data information and modify part of the system settings. In the guarantee does not affect the normal eating pig situation, the information detected by the sensor intake of pigs, the sensor node and gateway node to establish a communication mode, and connects to the computer through the serial port, real time monitoring of the health of eating pigs, to achieve the prediction pig disease. The system is capable of accurate measurement of intake of pigs, and the pigs through a wireless sensor network identification ID number, the unified management of the data network. Overall system block diagram shown in Figure 1. 


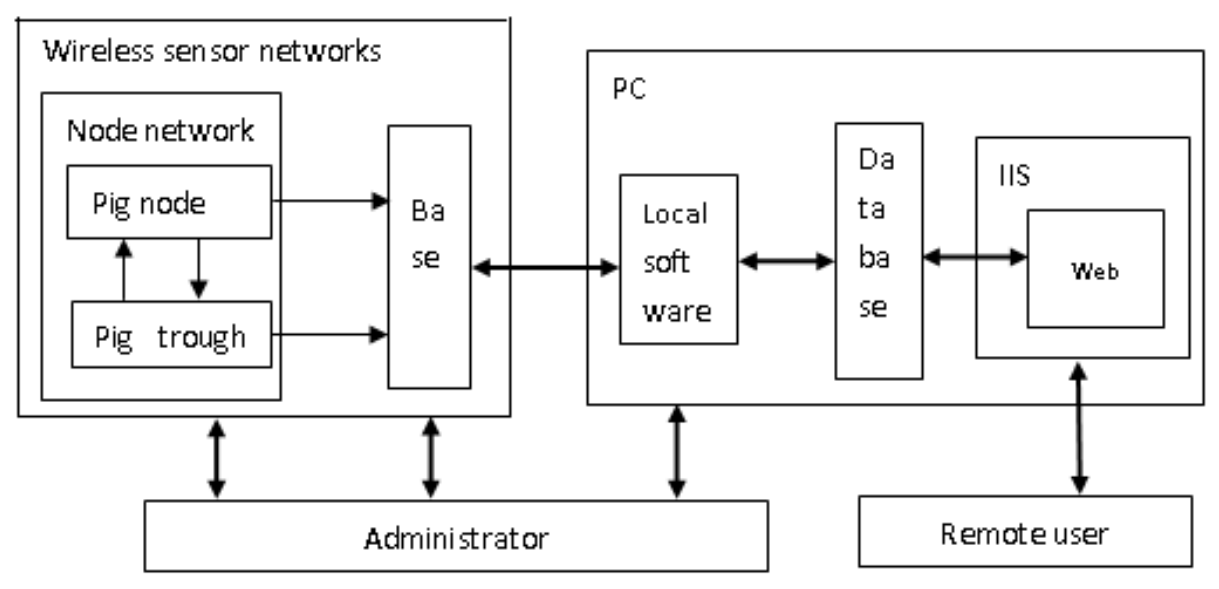

Fig. 1 Overall system block diagram

\section{Machinery}

Mechanical means in part by feeding port, bracket, manger, weighing sensors, wireless node, base station, USB to serial cable, PC machine, wherein the upper end of the trough by the load cell screw riveting, the load cell through the lower end of the bracket plate screws riveting, the wireless node via a signal line load sensor data acquisition, there is a gap between the trough and the bracket side plate, the load cell readings no systematic error, wireless node through the wireless sensor network to transmit data to the base station, the base station via USB to serial cable to transfer data to a PC, PC machine for data transmission station for recording, analysis and accurate measurement of food intake in pigs and pig through a wireless sensor network identification ID number, the unified management of the data network.

The system uses four load cells were placed in the four corners of the bottom of the trough, by screws with the upper trough riveting, weighing below the bottom sensor bracket by screws riveting, fixed trough this way and increase system stability. Manger side with the bracket side there is a gap, so as not to affect the weighing data. Eating pig trough near the same time, the subject of wireless nodes wake fournieri triggered by a low-power mode, into the normal operating mode, each feeding slot can only accommodate a pig eating pig eating until the end After the wireless node will load sensor before swine eating, after eating manger total weight transmitted to the base station through a wireless sensor network, the base station will receive the data via the serial port to send data to the PC, PC machine to the base station transmission record, analyze and accurately measure food intake in pigs and pig PC can recognize the ID number of the wireless sensor network, and then to determine the intake ID number corresponding pigs. Mechanical structure is shown in Figure 2.
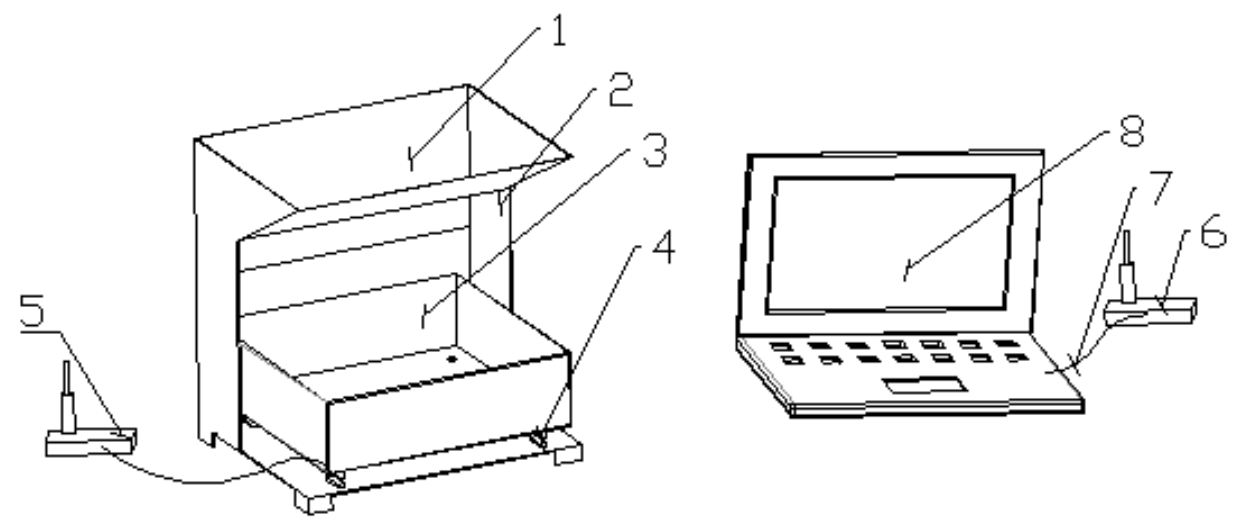

Figure 2 Pig feeding apparatus Chart 
As shown in Figure 1, the apparatus comprising: a feeding port 1, frame 2, manger 3, 4 weighing sensors, wireless node 5, base 6, USB to serial cable 7, PC machine 8.

Pig feeding trough near the trigger circuit, the circuit enters the normal operation mode; sensor before swine eating, after eating the total weight of the data transfer to the wireless node manager; wireless node through the wireless sensor network to transfer the data to the base station, the base station receives the data, data lost the PC, PC machine for data that is being analyzed by the serial port out through the wireless sensor network identification pig ID, records and pig food intake corresponding to the ID.

\section{Wireless Sensor Network Design}

TinyOS wireless sensor network operating system itself offers a range of components that can be very simple and convenient programming, to acquire and process sensor data and to transmit information via radio. When building TinyOS wireless sensor network, it will have a base console, is mainly used to control the various sensors child nodes, and they gather and process the information collected [3-6]. TinyOS simply issue in the console management information, then pass through a wireless network by each node to each other [7], and finally to concerted purpose, and convenient.

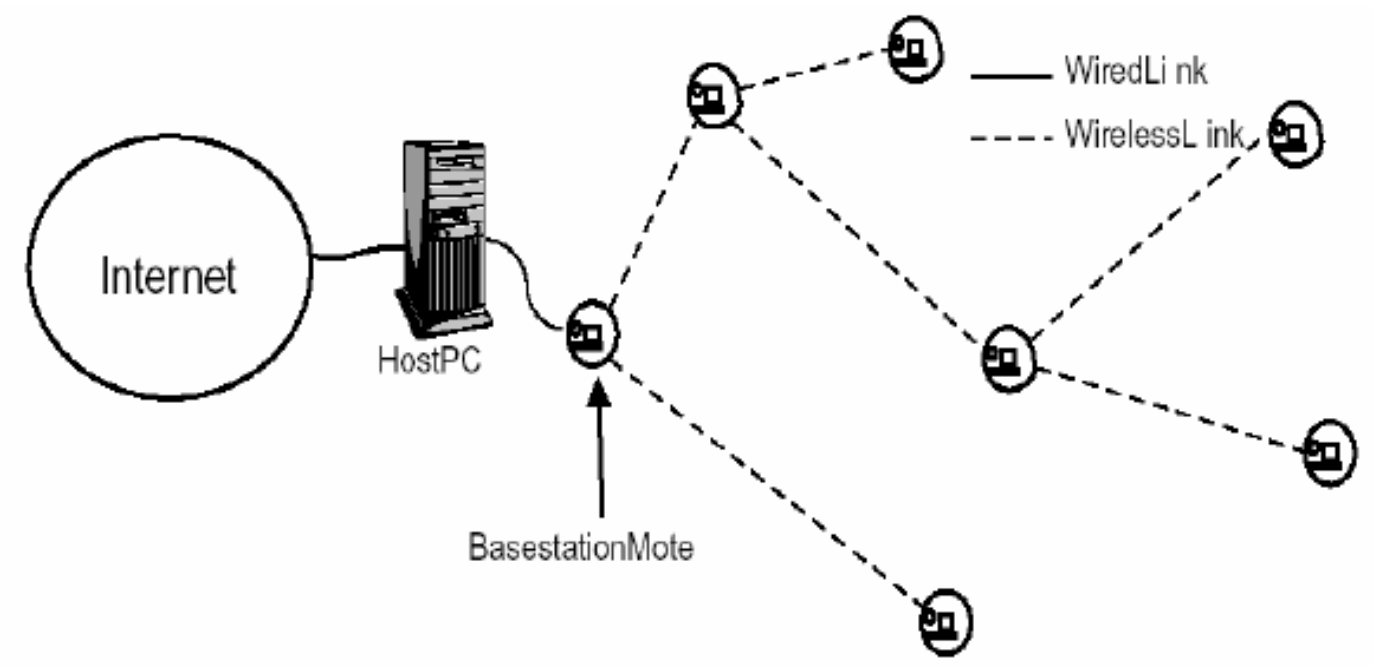

Figure 3 Wireless sensor networks based on TinyOS

Node Monitoring Section. This section needs to be laid sensor nodes, sensor nodes to access the wireless sensor network in the vicinity of the feeding trough. After starting base for system initialization, networking is set after initialization, if unsuccessful, repeat to networking success. After successfully building a network, enter the circulation, in turn determines whether a node joins, serial or sensor nodes request acquisition command response, if it is determined you need to perform, execute the stored sensor address respectively, the sensor sends acquisition command, monitoring data received to serial transmission . After the sensor node starts, system initialization, looking after the initialization and joins the network, if added to the cluster to repeat the success. After joining the network every five minutes to perform data acquisition cycle, then the completion of sending to the base station [8-9]. As shown, the oval pig sensor nodes and sensor base plate. 


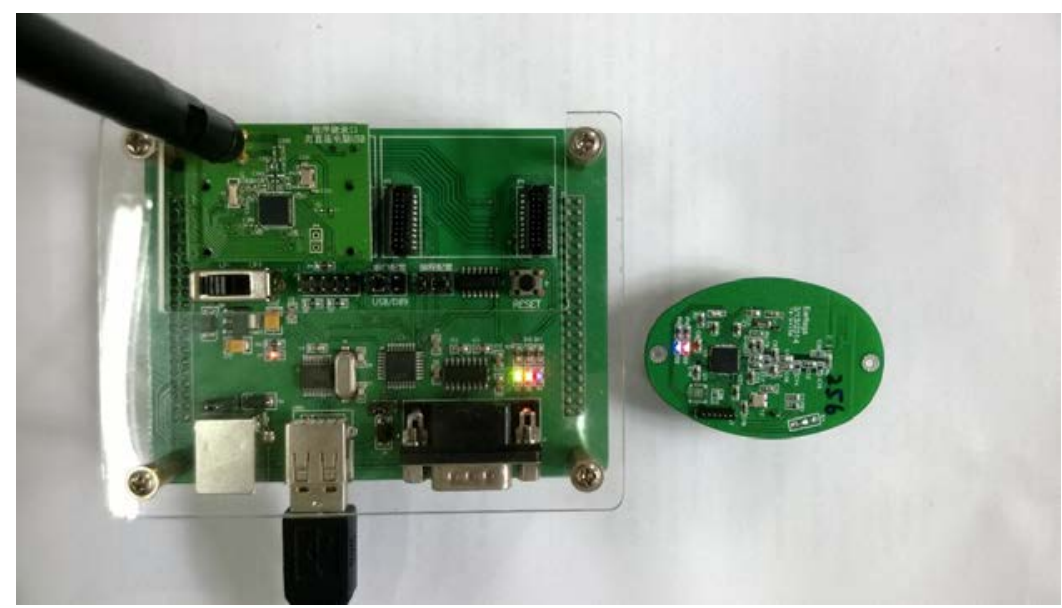

Fig. 4 Base stations and Node

RF circuit. RF module circuit is shown on the standard circuit RF chip CC2530 TI's recommended reference design made, CC2530 radio frequency module circuit diagram shown in Figure 3-3. To use unbalanced antenna, balun matching circuit is required to optimize the performance of the antenna circuit. Balun matching circuit cost can be used to achieve separation of inductors and capacitors, figure C8, C9, C10, C11, C12, C13, C14 and L1, L2, L3 constitute a balun RF matching network, the entire network Barron meet the RF input/output matching $50 \Omega$ resistor requirements. R6 is mainly used for the CC2530 provides an appropriate reference current, resistance is $56 \mathrm{~K}$. 32MHz quartz resonator $\mathrm{Y} 1$ and capacitors $\mathrm{C} 15$ and $\mathrm{C} 18(22 \mathrm{pF})$ constitutes a 32 $\mathrm{MHz}$ crystal oscillator circuit, $32.768 \mathrm{KHz}$ Y2 quartz resonator and capacitors C16 and C19 (15pF) constitutes $32.768 \mathrm{KHz}$ the crystal oscillator circuit, these two crystal oscillator circuit is main chip provides the appropriate clock. Furthermore, in order to further reduce the area of the entire sensor nodes, in accordance with the TI application documents Small Size 2.4 GHz PCB antenna, designed PCB inverted F-type antenna. In addition, in order to further improve the transmission distance between the wireless sensor node, in the realization of the board antenna while in the RF module interface section specially reserved for an external antenna.

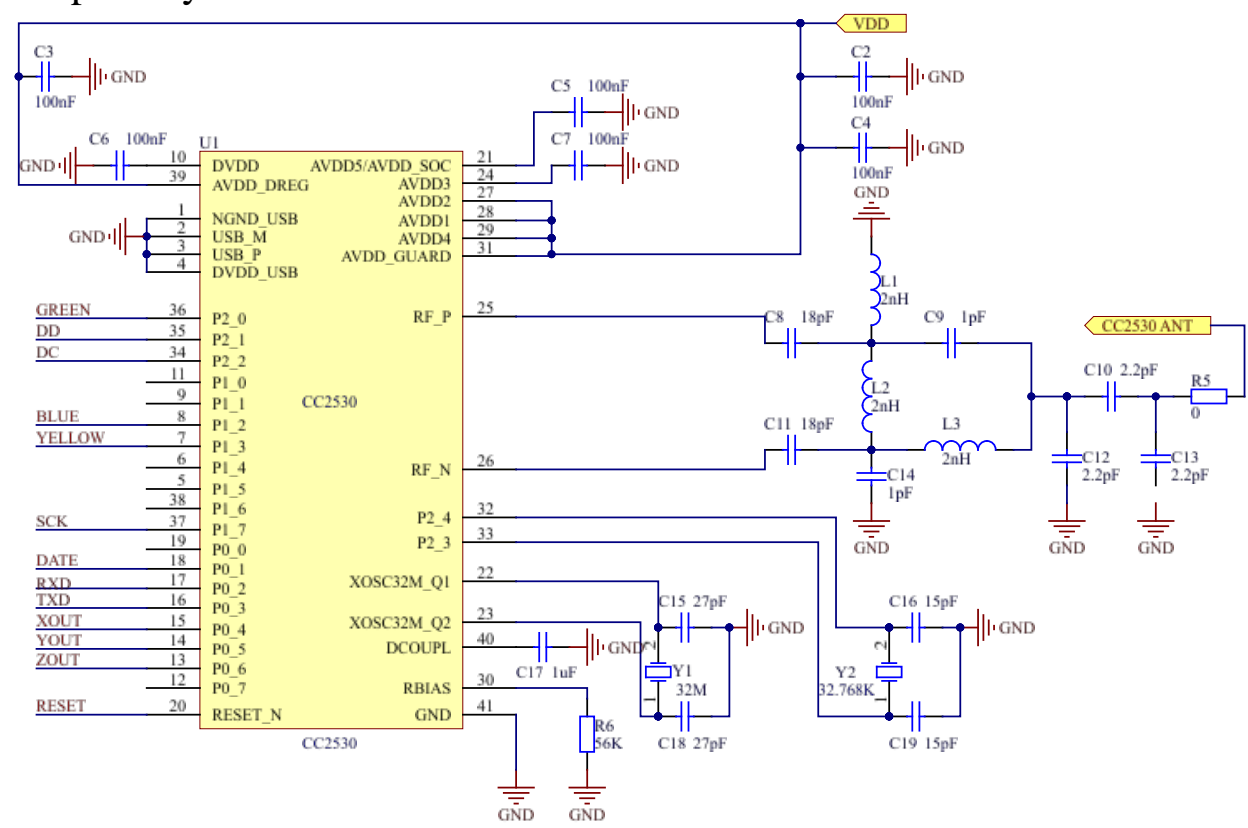

Fig. 5 RF circuit diagram

\section{The Interactions with Database of Upper System}

Whether it is local or network-side data analysis software keep all interactions with the client database in the initialization time it needs access to initialize the sensor configuration table for the resolution of the sensor configuration of serial data packet collection. When the data analysis is 
complete, we need to node data stored in the database so that users can view the network dynamic data curve or historical data. In addition, the sensor node configuration and sensor type configuration are deployed in the network, giving users a very friendly management interface, while allowing remote system configuration can be modified to facilitate the management of personnel management.

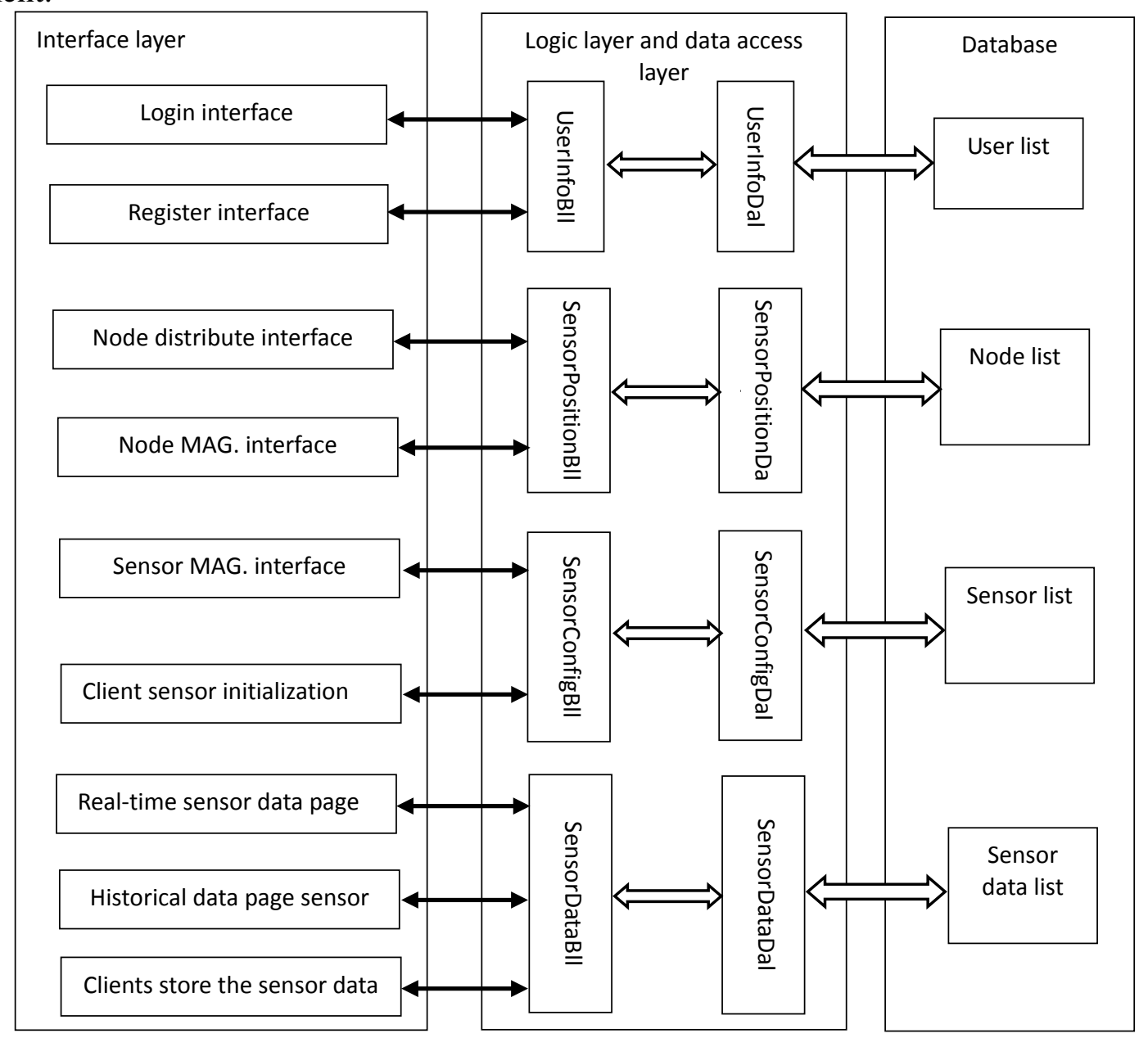

Fig. 6 System and database diagram

\section{PC Part of the Design}

Client programs written using C\# language, C\# is Microsoft's .NET framework-based, object-oriented high-level programming language. $\mathrm{C \#}$ from $\mathrm{C}$ and $\mathrm{C}++$ language is derived from inherited its strong performance, but again based on the .NET Framework class library, with a high rapid development capabilities. C\#"is simple, modern and universal" design principles, and strong type checking, automatic garbage collection and other features make C\# is very easy to use and has a strong programming productivity.

Microsoft launched a database using a relational database solutions Microsoft SQL Server 2008 R2. The platform not only supports graphical interface operation, while supporting SQL database direct CRUD, greatly reducing the time and cost of developing and managing the data infrastructure. In addition, the platform also has a very high security, reliability, scalability, so users only need to worry about implement applications. Database can be convenient to use the sensor data storage, analysis, management, and sharing.

\section{Conclusion}

For the overall consideration of traditional pig industry and future intelligent pig detection system, this paper designed pig food intake measurement system based on TinyOS and wireless sensor networks. The system is low cost, easy to operate, etc., and has good portability and it can be used to monitor a wide range of farming. The system not only avoids too much of the intensity 
labor of the people, improve the environment, farm equipment, and improve production efficiency. It scientific analysis and observe the health of pigs, thereby effectively preventing disease. It has great significance for the development of the intelligent pig and provides some new solutions for farms modernized monitoring system.

\section{Acknowledgements}

The Fundamental Research Funds for the Central Universities, under grant project 2013PY052

\section{References}

[1] Liang Iraq, Huang Sixiu. Domestic and Livestock Industry Development Situation and Trends of South China Agricultural University, 2007(1): 50-53.

[2] Xu-Jing D, Zhao-Wen Y, Miao Li. Other design-based ZigBee Wireless Sensor Network. Computer Engineering, 2010, 36 (10): 110-112.

[3] Qi Nan, Han Bo, Li Ping. Smart Home ZigBee Wireless Sensor Networks Based Design. Electrical and Mechanical Engineering, 2007, 24 (2): 20-22.

[4] Yin Hang, Zhang-Qi S, Cheng-Zhi L. Temperature and Humidity Monitoring System Based on ZigBee Wireless Network. Electrical and Mechanical Engineering, 2008, 25 (11): 20-23.

[5] Ma-Zhu C, Sun-Yi N, Mei Tao. Summary Wireless Sensor Networks. Communications. 2004 (4).

[6] Si-Hai F, Yang Zhong, Wang Jun. Research Status and Application of Wireless Sensor Networks. Electrical Engineering, 2011, 28 (1): 16-20.

[7] Pan Hao. Wireless Sensor Network Operating System TinyOS.

[8] Zhu Jin, Yang-Zhan Y. Design based CC2530 Wireless Vibration Monitoring Sensor Nodes. Instrument Technique and Sensor, 2012 (8): 56-58.

[9] Li-Wai Y. CC2530 and Wireless Sensor Network Operating System TinyOS Application Practice.

[10] Xiao-Su Q, Han-Hou Y. Flow and Application Guide. Beijing. China Petrochemical Press, 1999. 